SESSION 2632

\title{
Context Based Educational Java Applets Using Consumer Products
}

\author{
Alexander N. Cartwright, Pratibha Gopalam, \\ N. Liu, Z. Yuan, T. Tang and Chu R.Wie \\ Department of Electrical Engineering \\ Center for Active Learning of Microelectronics and Photonics \\ State University of New York at Buffalo \\ Buffalo, New York 14260 \\ E-mail: anc@eng.buffalo.edu, wie@eng.buffalo.edu
}

\begin{abstract}
The popularity of the Java language for educational materials development continues to increase. Unfortunately, most of these developed tools are isolated Java applets that explain a single "textbook" educational concept. These disjoint Java applets, while sometimes providing excellent educational materials, lack the system level, top-down approach that is prevalent in engineering. In this paper, we present our work on developing Java applets, and web-based courseware materials, for microelectronics and photonics (lasers and optics) that incorporates this system-level top-down approach. Specifically, we will discuss the use of consumer products, such as barcode scanners and CD-ROM's, as effective microelectronic and photonic systems learning modules.
\end{abstract}

The implementation of this top-down consumer product approach requires that the developer understand the applications of these theoretical and educational materials. In this way, the developed web-based courseware provides excellent engineering educational value by providing instructors and students with a context for the specific topic. That is, the consumer product approach effectively provides a link from the study of microelectronics and photonics to real world applications.

A user of the courseware enters at a high level view of an everyday real-world consumer product, selects components of that product to investigate, and can interact with the Java applets that describe the details of the operation of the selected component. Moreover, the developed web-based courseware allows the user to choose the level of educational materials that he or she wishes to study. In this way, these educational materials become accessible to users with educational backgrounds varying from high-school students, with limited science experiences, to the researchers in the fields of microelectronics and photonics. In this paper, the overall "big picture" implementation issues as well as the design of the applets embedded within this topdown consumer product approach are presented. 


\section{Introduction}

The development of the Java programming language by Sun Microsystems provided a new tool for the development of interactive web-based educational materials. Specifically, the ability to develop Java applets that can be instantly shared on the web in a platform independent manner has permitted the developers of these applets to reach large target audiences. The visual interactive simulation of technical subjects using Java applet programs on the World Wide Web (WWW) has a proven effectiveness for students learning difficult subjects. For example, we have found that employing Java Applet simulations in a junior-level university course has shown both qualitative benefits and quantitative benefits in student quiz and grade performance [1,2].

In spite of the benefits that are provided by these educational applets, there still exist many impediments that need to be overcome before their full potential as educational aids can be realized. The necessity of presenting these applets with relevant courseware, the slow speed of web access to these applets, and the time-intensive development efforts required for these applets are three of the most important impediments that need to be considered. Moreover, most of the already developed Java applets, even those that provide excellent educational materials, lack the system level, top-down, approach that is prevalent in engineering. More importantly, most of the already developed educational applets target a specific educational level.

In this paper, we present our work on developing Java applets, and web-based courseware materials, for microelectronics and photonics (lasers and optics) that incorporates a system-level top-down approach. We discuss the pedagogy associated with using these materials, provide examples of the implementation, and provide a detailed design procedure for the component applets. A user of our courseware enters at a high level view of an everyday real-world consumer product (see Figure 2, for example), selects components of that product to investigate, and interacts with specific Java applets that describe the details of the component operation. Furthermore, users of this web-based courseware are allowed to choose the level of the educational materials that he or she wishes to study. In this way, these educational materials become accessible to users with educational backgrounds varying from high-school students, with limited science experiences, through the researchers in the fields of microelectronics and photonics. Finally, the problems associated with the slow speed of accessing these applets through the web, and with the time intensive development required for these applets, are addressed in the discussion of the design and development phases of our applets.

\section{Courseware Pedagogy}

Figure 1 shows a graph of the Java courseware pedagogy with an example implementation. As shown in this figure, we choose a ubiquitous consumer optoelectronic product to serve as the entry point in our case-study module. This case-study module is used as the motivation for the topics to be considered. In addition, included in the top-level case-study module is the ability for the user to choose their desired learning level for the topics. Once an educational level is chosen (A, B, or C in Figure 1), the interactivity of the applet is adjusted for that user level. Specifically, in our implementation, if the user chooses educational level A, the menus that popup on mouseover events are only those associated with that educational level. Another benefit of this layout is that once the user chooses the level, the component applets and 

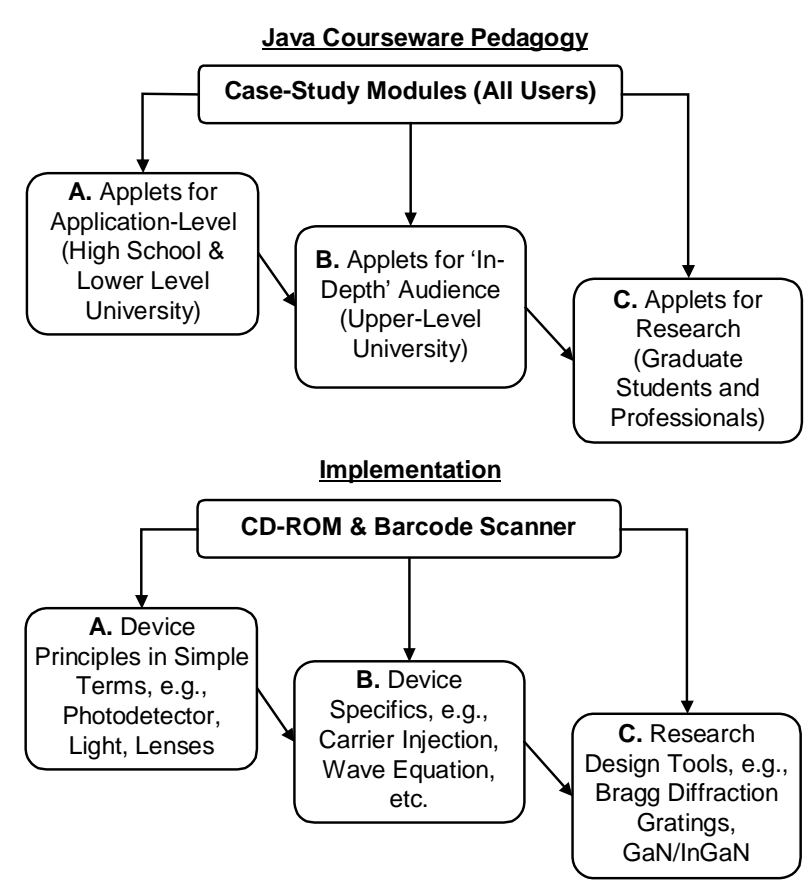

Figure 1: Java courseware organizational structure with example implementation. associated educational materials for all other educational levels are hidden. The menu options that appear for components are links to the underlying Java applets that focus on component explanations appropriate to the users chosen educational level.

These case study modules, and the underlying educational applets, can be seamlessly integrated into a number of course syllabi that include the study of semiconductors, photonics, lasers and optics. In fact, all of the associated component applets are developed as educational aids for specific textbook style problems (see Figure 3 and Figure 4 for example) for students in traditional courses and can be readily linked to topics studied in a textbook. The main advantage of the top-down module level approach is that these top-level consumer product applets can serve as stand alone, selflearning modules.

A well-chosen case-study module can provide a rather comprehensive set of topical concepts that could fill a substantial part of a semester-long course. In addition, discussions of consumer products engage the students with the subject area and legitimize the theoretical aspects of the course. Some of the important criteria for selecting the case study modules are

1. The various components of the product should provide examples of the devices/concepts that we want to explain (in our case the product must include semiconductor and photonic devices).

2. The product must have a challenging intellectual appeal.

3. The product should be attractive enough for inclusion in University and high school courses.

Once an appropriate consumer product is chosen, the case studies of these products are implemented as applets. A complete case study requires the development of a number of independent complex applets to describe the various components of the top-level system. We have found that these components applets are best served by including them in a detailed html page. This html page includes theoretical details, links to other educational resources related to this topic, and other non-animated graphics and photographs that are tailored to the appropriate audience level. The links to other educational resources expand the usefulness of our developed resource. 
The snapshot of the barcode scanner applet implementing the barcode scanner case study is shown in Figure 2. The menu options at the bottom of the figure allow the user to choose the educational level. In addition, the user can move the mouse over the image and level appropriate menus appear that show developed component materials. In the figure, we show the menu for the mouse passing over the laser beam. The barcode scanner fulfills the three criteria given above for an excellent case study module. Specifically, the barcode scanner provides sufficient components for entry into microelectronics and photonics: a laser (He:Ne or semiconductor laser diode), optics for scanning and a detection system (either a photodiode or a CCD array), circuitry for detection, etc. Moreover, its prevalence throughout society (used in stores to scan the UPC code of products into a computer for inventory and pricing) makes it recognizable to many people. Barcode scanners use the absorptive behavior of black versus white to provide the zeros and ones necessary for the digital encoding of the product identification and provide us with a mechanism to introduce the electronics/optics interfacing. Specifically, once the laser scans across the barcode, consisting of black and white stripes, it either sees regions of high absorption and low scattering of the laser (black regions) or regions of low absorption and high scattering of the laser (white regions). The detection system records the scattered signal as a function of time

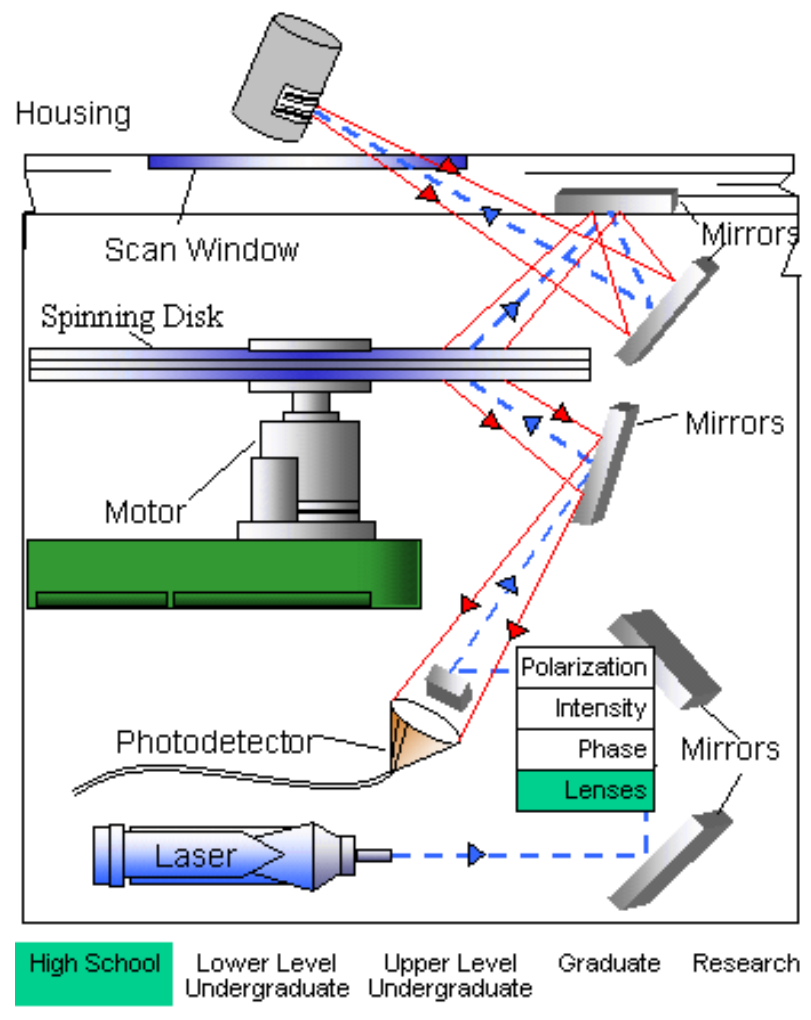

Figure 2: Barcode Scanner Case-Study Module. The user is allowed to change the menu at the bottom, and to select from pop-up menus that appear as the mouse moves over the applet. The menu shown is for light propagation and appears when the mouse passes over the laser beam. (as the laser scans across the barcode). The signal on the photodetector will be proportional to the amount of light it receives and thus we can record either lows or highs using this system.

As stated earlier, users are allowed to select any component of this product. For example, by selecting the laser we can go to the component applet on either semiconductor lasers or to a component applet on gaseous lasers such as He:Ne. In the barcode scanner case study, menus appear when the user moves over the barcode, holographic plate, laser, detector, mirror, laser beam, etc.

The top-level applets (case study modules) serve a dual purpose. For students with an already existing interest in the science, mathematics, engineering and technology (SMET) area, they serve as an initial entry point for the more in-depth applets and investigation. For people without an existing interest in the SMET area, the toplevel applets can serve as one of the main learning vehicles for entry into the exciting 


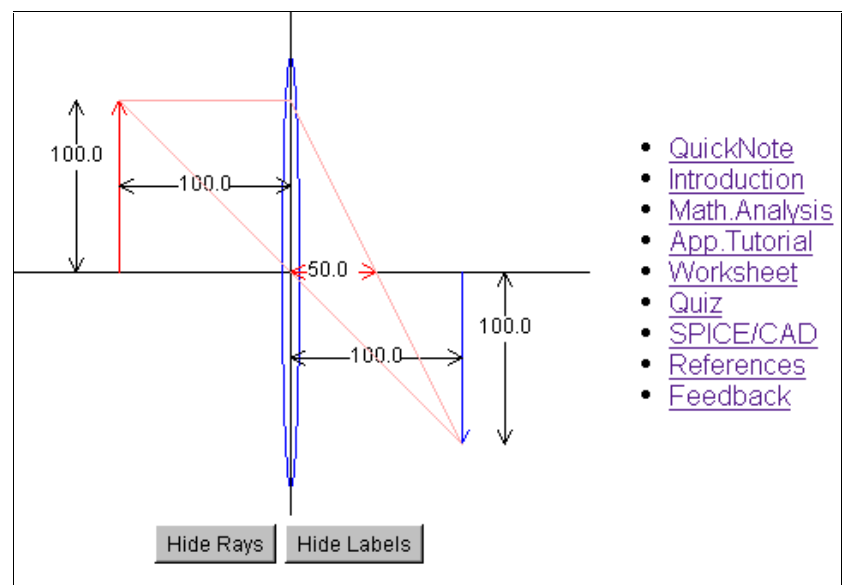

Figure 3: Example component description applet. This component applet describes the behavior of a lens system and is intended for the High School and Undergraduate Levels. The links of the right side provide worksheets, quizzes, mathematics and feedback options. world of technology and their underlying scientific principles.

As stated earlier, it is essential to develop all the component applets for a case study so that the entire module is appropriate for problem-based learning [3]. In this way, these modules 'naturally' induce such questions as to how the product works and what components are necessary to enable its functionality. These case studies introduce an element of reverse-engineering by allowing learners to dissect a product, 'open up' each component to investigate the governing scientific principles, and to explore effects produced by varying 'physical' or 'structural' conditions (all accomplished by pressing a key on the keyboard or clicking the mouse). Therefore,

for each developed module we develop component applets that serve as stand-alone simulation tools for the basic concepts as well as constituent parts for the case-study modules. More importantly, associated with each component applet, we develop the pedagogically important peripheral materials such as images, texts, equations/theories, applet worksheets, quiz questions, and tutorials to complete the educational experience.

\section{Component Applets}

The developed component applets must be appropriate for the intended educational level. In our model, applets for the lower level should be appropriate for high school seniors and low-level university students. Applets that describe the operation of devices in a 'black-box' manner are appropriate for this level. For example, Figure 3 shows a developed applet that illustrates the imaging of an object. This applet allows the user to independently change the focal length of the lens, and the height and position of the object and image. In this way, the user can perform the 'research' to develop the lens equation.

As the educational level increases, the component applets must increase in complexity and must include the details required to stimulate the advanced learner. For example, in photonics, applets that describe light propagation using ray matrices (ABCD matrices) are being designed for upper-level undergraduate students. In addition, applets that include more details of laser operation, such as cavity design and stability, laser dynamics (both for semiconductor laser diodes and conventional lasers), and introduce newer materials for the next generation of blue lasers (such and $\mathrm{GaN} / \mathrm{InGaN}$ based lasers) are appropriate for the graduate and research level users.

At the present time, our efforts have focused on the development of applets suitable for the high school and undergraduate levels. Specifically, we have focused on the development of applets 
that can be easily incorporated into undergraduate courses and high-school courses to have a much larger impact through a broadened dissemination. In the following section, we discuss the design and development criteria that are followed in the software development cycle of the educational applets used in these materials.

\section{Educational Applets design and development}

The design (and analysis) of an applet mainly involves the design of the applet screen and the design of the various visual components of the applet in conformance to a set of general but important guidelines that usually improve a user's perception of the problem [4]. The development strategy mainly focuses on the structured software development process model followed to reduce the time and effort involved in developing a new applet. Here, we present the general guidelines for the design and development of these applets.

\section{General Guidelines for Applet Design and Development}

Applets catering to a wide audience should be simple in terms of the subject content and the screen content. They should have good visual representation of the important scientific concepts. The applets should allow the full range of user interactivity with a bi-directional design, presenting the output condition as a response to a changing input condition and vice versa. The technical content of these applets must be accurate. Applets that perform extensive calculations should use fast computational algorithms to allow "real-time" update of the applet output in response to user input. Using appropriate computational algorithms helps to solve the speed issue mentioned earlier.

\section{Screen (Content) Design and Analysis of Use Scenarios}

The first, and perhaps the most difficult, task is the design of the applet screen. After the applet topic is thoroughly analyzed, the overall screen content should be designed and sketched in a notebook. Based on this initial sketch and based on pedagogical considerations, all 'possible' use-scenarios are then enumerated. A brief description should be provided for each scenario. These scenarios are then implemented as user interaction scenarios in the development phase.

\section{Design of Visual Components of the Applet: Object-Oriented Design and Implementation}

In the design of the visual components, the applet screen is analyzed according to object-oriented programming principles like encapsulation by classes, abstraction through interfacing and code reusability by inheritance. Encapsulation of different visual components in classes, and abstraction of these components by providing interfaces through public methods in the classes, is essential for future enhancements and maintenance of the applets.

Educational applets should demonstrate a single educational topic. Therefore, it is good practice to encapsulate all the underlying theories and formulae in a single Java class. All theoretical equations, formulae and variables, along with solution algorithms, are placed in this class. In this way, all theory-related problems can be corrected by correcting this class. Moreover, there must be a single instance of this class in the applet. One approach to ensure that the applet has 


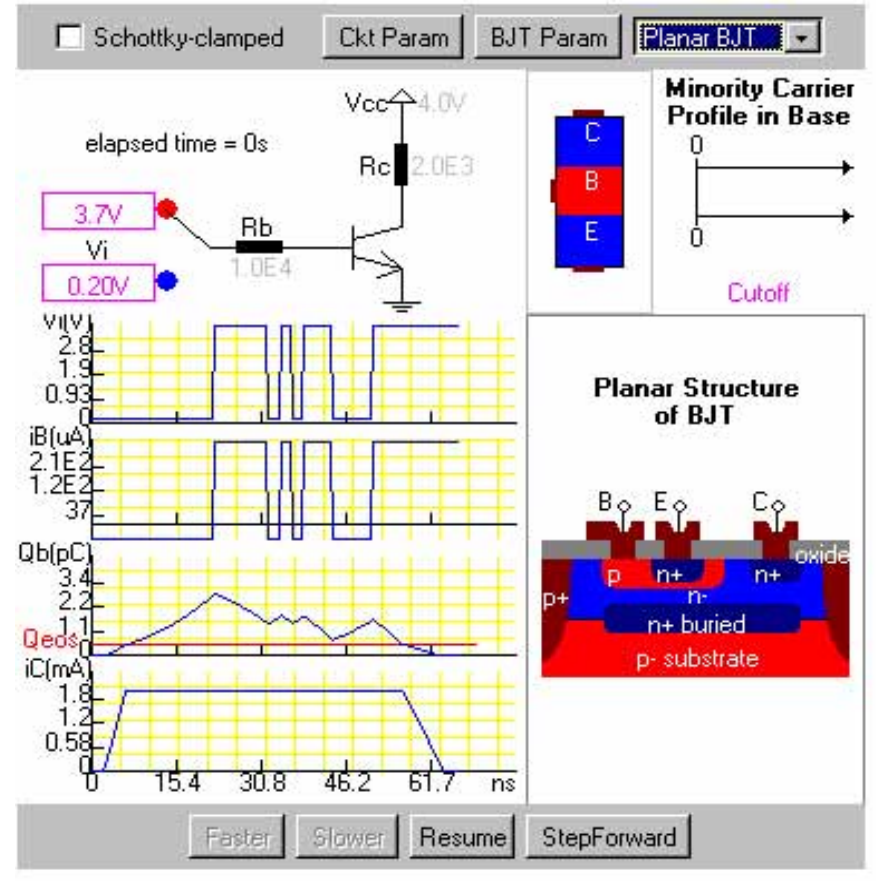

Figure 4: This applet has been developed following the software development model described in this paper. Several distinct graphic components comprise this applet and each graphic component was refined separately. only one instance of this 'theory' class is to declare all member variables and methods as static. An alternate approach to this design, which encourages the design of a generic architecture for visual simulation, is to follow the Singleton design pattern for the 'theory' class. For reference, the book by Gamma et al. [5] discusses this Singleton design pattern.

\section{Development by structured software development process}

A structured software development process facilitates the development of graphically-oriented Java applet programs. Employing such a development model provides a resourcesharing environment for group collaboration environments. For example, some class files may be shared among the developers as a reusable library class or as part of a single applet program developed collaboratively. This puts these class files through rigorous

test cycles when they are repeatedly used in different case scenarios and by different users.

In addition to code reuse, structured development of visual components of the applet breaks the applet into smaller components that can be easily developed and tested. Moreover, the project supervisor does not have to wait until the entire program is completed in order to examine the initial output. This process is of great benefit when the applet is developed by a student assistant supervised by a college professor. The professor is becomes responsible for the educational content and the pedagogical aspects while the student programmer is responsible for all programming issues.

\section{Design and Development: An educational Java Applet on Bipolar Transistor switching process}

The snapshot of the applet illustrating a Bipolar Transistor switching process is shown in Figure 4. This applet provides a visual simulation of the switching characteristics of a bipolar junction transistor. The objectives of this applet are to demonstrate the BJT switching process, to illustrate various physical processes responsible for the time delays, and to illustrate the effect of a Schottky-diode clamp. The design of the applet begins with a sketch of the entire screen view of the applet program. This initial sketch comes after a considerable amount of study, analysis and design of the subject topic to be simulated. This sketch determines the steps to follow in the software design and analysis. This initial sketch is an overall view of the program and takes into 
consideration the description or presentation of the problem, the detailed user interaction and control scenarios, and the visual presentation of the simulation result.

The bipolar transistor switching process is represented by three major graphical components in this applet:

1. The diagram of the RTL circuit where the user can toggle the input voltage between high (default, $3.7 \mathrm{~V}$ ) and low (default, $0.2 \mathrm{~V}$ ) by a mouse click.

2. The graph of waveforms, which, in response to low-to-high or high-to-low input switch, plots various voltage and current signals as a function of the elapsed time since the last switching. The specific parameters plotted are the input voltage (Vi), the input Base current (iB), the excess minority carrier charge in the Base $(\mathrm{Qb})$, and the Collector current (iC).

3. The excess minority carrier profile in the Base region. This plot shows the excess electron concentration profile in the Base of an npn transistor.The spatial dimension of the Base region matches the Base width of cross-sectional diagram .

The switching time delay is dynamically drawn in the waveform graphs. Physical processes responsible for the time delay are shown in the plot of excess minority carrier profile in the Base and the plot of the space charge region of the Emitter-Base junction. The EB junction is shown in the lower-right portion of the applet screen when the "EB Junction" is selected in the choice box on the top 'menu bar' of the applet. This EB junction plot shows the junction charging process during the turn-on.

The effect on the transistor turn-off delay time due to the placing of a Schottky-diode clamp at the Base-Collector junction is demonstrated in all five graphic components of the applet. Placing a Schottky diode in parallel with the Base-Collector junction is represented by the contact metal to the Base extending into the Collector region in both the BJT cross-section and the planar BJT diagram, and by the diode symbol connecting the Base and Collector terminals in the RTL circuit diagram. The effect of Schottky-clamp is shown in the excess minority carrier plot and in the waveform plot.

This applet program embodies a single subject topic or content that is common throughout the entire applet. Each development unit consists of common files. The files for this example applet are as shown in Figure 5. The files follow these general guidelines:

1. Test $\langle$ Unit>. html : This is the html page to view the applet by a web browser or by the appletviewer program from Sun Microsystems, inc.

2. <ComponentUnit>.java: This class is the main class that implements the graphical drawing and the behavior of a particular unit. It is usually problem-specific and depends on Formula.java and the other problem-independent Library classes (Java core or custom library). 
3. Test $<$ Unit $>$ Applet.java: This applet is a dummy applet class, just to test the ComponentUnit class.

The file Formula.java encapsulates the theory. The variables in Formula.java have a static scope so that there can be only one value throughout the applet program. All the problem-specific component units of the applet depend on this Formula.java. Furthermore, each screen component is developed in a separate refinement scope (development unit). In this example, the five main screen components are the circuit diagram, the BJT transistor schematic, the excess minority carrier profile in the Base, the waveforms and the planar BJT structure

During the initial analysis of the component unit, objects with a general or reusable property are identified. These objects are implemented into reusable custom library classes. For example, from the development of the Resistor-Transistor logic circuit, we have designed many circuit reusable symbol classes into generic library classes for use in other applet projects. These classes are then documented and included into our semiconductor Java class library [6]. After merging all the component unit class files into a single directory, the applet class that implements the overall simulation of the problem is developed. This applet accomplishd the simulation of the entire problem by using several (five in the above example) <ComponentUnit>.java files from each of the refinement scopes. In this class, a complete range of user interactions using graphical user interface must be implemented.

\section{Evaluation}

We are constantly in the process of using these modules in courses. For example, we are testing some of the recently developed photonics applets in a senior level undergraduate course, EE492 Lasers and Photonics. The evaluation is mostly based on student responses and feedback. In this work we are focusing on providing the developed educational applets with the necessary context to stimulate student interest. At the time of writing this paper, there is insufficient feedback to accurately evaluate the effectiveness of this approach. We have previously developed a number of applets that have been used in a number of different courses at the University at Buffalo and at other schools throughout the world.

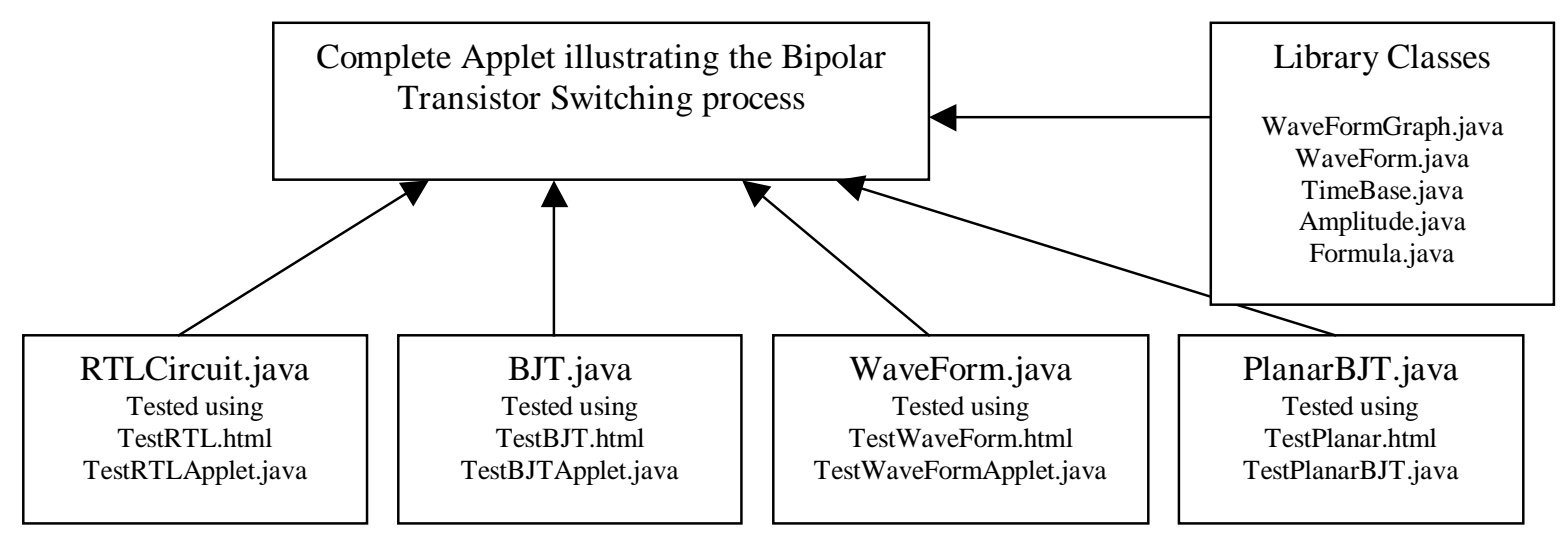

Figure 5: Example files for the BJT Switching process applet. Each component is tested individually and then as a complete set. 


\section{Conclusions and discussions}

We are currently developing and evaluating Java applets and associated materials for learning/teaching semiconductor and photonic systems, devices, processes and concepts based on ubiquitous consumer products. Most people use some high-tech' product on a daily basis that have embedded semiconductor or photonic devices and integrated circuits. Unfortunately, most people do not realize engineering and physics that comprises these devices. The develop resource will provide essential teaching and learning materials that can be used at various levels of education and provide insight into how consumer optoelectronic devices work. The ultimate goal of this work is to stimulate students to enter the exciting fields within science and engineering.

\section{Acknowledgments}

The authors would like to acknowledge the support of the National Science Foundation Award \#9950794 for the support of this work. We also would like to acknowledge the contributions of Xin Hu, Derek Hoiem and Philip Manijak in the development of the educational applets.

\section{Bibliography}

1. Wie, C.R., "Educational Java Applets in Solid State Materials" <invited paper>, IEEE Trans. on Education

Vol.41, No.4, November 1998. In press. http://wmm.coe.ttu.edu/ieee trans_ed/nov98/BEGIN.HTM.

2 Montgomery, S. M. "Addressing Diverse Learning Styles Through the Use of Multimedia."

http://FrE.www.ecn.purdue.edu/FrE/asee/fie95/3a2/3a22/3a22.htm.

3. C.K. Chiklis, "Engineering Design in Materials Science and Engineering: A Model for University-Industry

Cooperation, Part II.” J. Mater. Edu. 19 (1997) 145-154.

4."Educational Java Applets: Object-Oriented Development For Reuse And Maintenance", C.R.Wie, Nanzhu Liu and Z. Yuan, International Conference On Simulation And Multimedia In Engineering Education, 2000 Western MultiConference, JANUARY 23-27, 2000 San Diego, California.

5. Gamma, E., R. Helm, R. Johnson, and J. Vlissides. 1995. "Design Patterns: Elements of Reusable ObjectOriented Software," Addison Wesley Longman, Inc.

6 http://jas2.eng.buffalo.edu/semiconductor/apiWIE/packages.html.

ALEXANDER N. CARTWRIGHT: Alexander N. Cartwright received his Ph.D. from the University of Iowa in 1995 and has been an Assistant Professor at the State University of New York at Buffalo since August 1995. In 1998, he received a NSF CAREER Award that supports his research on GaN based optoelectronic devices and his educational activities. He is Associate Director of the Center for Active Learning of Microelectronics and Photonics.

PRATIBHA GOPALAM : Pratibha Gopalam is a graduate student in the Electrical Engineering Department at the State University of New York at Buffalo. She is a member of the group developing educational Java applets at the Center for Active Learning of Microelectronics and Photonics. She received her undergraduate degree in Electronics and Communication Engineering from Bangalore University, India, in 1997. She worked as a software engineer for Hewlett Packard India Software Operations and Asea Brown Boveri Pvt. Ltd. before joining SUNY at Buffalo.

NANZHU LIU: Nanzhu Liu is a graduate student in the Electrical Engineering Department at the State University of New York at Buffalo. She is a member of the group developing educational Java applets at the Center for Active Learning of Microelectronics and Photonics. She received her BS in Physics from Xiamen University, China in 1995. In 1998, she got her M.S. degree in Condensed Matter Physics from Chinese Academy of Science, China.

ZHIYONG YUAN: Zhiyong Yuan is a graduate student in the Department of Electrical Engineering at the State University of New York at Buffalo. Presently he is a member of the group developing educational Java applets at 
the Center for Active Learning of Microelectronics and Photonics. He received his undergraduate degree from Zhejiang University and his MS degree from Harbin Institute of Technology in China.

TAO TANG: Tao Tang is a graduate student in the Electrical Engineering Department at the State University of New York at Buffalo. As a member of Center for Active Learning of Microelectronics and Photonics, he is working on educational Java applets development and device simulation with FLOODS/FLOOPS. He received a BS degree from Nankai University, China in 1996.

CHU RYANG WIE: Chu Ryang Wie received his Ph.D. from California Institute of Technology in 1985 and has been with SUNY at Buffalo ever since, and currently is a Full Professor. He was a Presidential Young Investigator awardee of NSF in 1988. The semiconductor Java applet simulation website, he and his students have developed, (http://jas2.eng.buffalo.edu) is internationally recognized as a most effective and valuable resource for microelectronics education. He is the Director of Center for Active-learning of Microelectronics and Photonics, and is the Program Chair of the International Conference on Simulation and Multimedia in Engineering Education, San Diego, CA, Jan. 23-27, 2000. 